\title{
LA PRODUCCIÓN DE MANUSCRITOS ILUMINADOS EN LA CURIA PAPAL A FINALES DEL SIGLO XIII: DOS NUEVOS PONTIFICALES CONSERVADOS EN ESPAÑA
}

\author{
MERCEDES LóPEZ-MAYÁN \\ Universidad de Santiago de Compostela
}

\begin{abstract}
En los últimos años se han llevado a cabo numerosos estudios sobre la miniatura producida en Roma durante los siglos medievales y, en relación con ello, se han tratado de recomponer los fondos librarios pontificios, disgregados entre bibliotecas de todo el mundo. El objetivo de este artículo es añadir a la nómina de los ejemplares ya conocidos dos pontificales conservados en Sevilla y Tui, pues, aunque hasta ahora nunca se habían relacionado con ese contexto, el análisis de su iluminación y de otros elementos demuestra que fueron producidos en la Curia papal a finales del siglo XIII.
\end{abstract}

Palabras clave: Pontifical; Miniatura; Edad Media; Curia papal; Sevilla; Tui.

THE PRODUCTION OF ILLUMINATED MANUSCRIPTS IN THE PAPAL CURIA AT THE END OF THE $13^{\text {TH }}$ CENTURY: TWO NEW PONTIFICALS PRESERVED IN SPAIN

In recent years numerous studies have been carried out on the miniatures produced in Rome during the Middle Ages, and in relation to them, an attempt has been made to locate pontifical books dispersed in libraries throughout the world. The purpose of this article is to add to the list of acknowledged examples two pontificals preserved in Seville and Tui which until now have not been related to a Roman context. Yet the analysis of their illuminations and other elements demonstrate that they were produced in the papal Curia at the end of the $13^{\text {th }}$ century.

Key words: Pontifical; Miniature; Middle Ages; Papal Curia; Seville; Tui.

En un breve artículo publicado en 1985 Valentino Pace denunciaba la escasa atención que los estudiosos habían concedido al sin embargo importante capítulo de la miniatura realizada en Roma en el siglo XIII ${ }^{1}$. El autor atribuía dicha situación a dos razones principales: la focalización del interés de los investigadores en las grandes obras monumentales de la época, en detrimento de hechos artísticos menores como la miniatura, y la dificultad de establecer los rasgos definito-

1 PACE, 1985. 
rios de esa producción manuscrita y, por tanto, de delimitar un corpus de obras indudablemente atribuibles a la actividad libresca romana. A ello habría que añadir la escasez y el carácter fragmentario de la documentación conservada sobre la biblioteca papal ${ }^{2}$ y la dispersión geográfica de sus fondos durante la baja Edad Media y los siglos posteriores ${ }^{3}$. Finalmente, la existencia de otros focos italianos de producción miniada de rasgos más definidos, como Bolonia o la región de Umbría ${ }^{4}$, hizo que el territorio romano quedara relegado a un segundo plano. Por esta razón, y en coherencia con la reflexión de Valentino Pace, a mediados de los ochenta empezaron a sucederse una serie de estudios con los que diversos investigadores contribuyeron, progresivamente, a perfilar los rasgos característicos de la miniatura producida en Roma al situar en la ciudad papal el origen de determinados manuscritos hasta entonces atribuidos a otros talleres italianos ${ }^{5}$. Daba, así, comienzo una línea de estudio que se ha revelado enormemente fructífera y que en la actualidad continúa produciendo interesantes trabajos que incrementan, día a día, el corpus de códices procedentes de Roma ${ }^{6}$.

La importancia de la producción manuscrita de dicha ciudad a lo largo del siglo XIII debe relacionarse con el contexto de reforma religiosa y de potenciación ideológica que protagonizó el papado, sobre todo a instancias de Inocencio III (1198-1216) e Inocencio IV (1243-1254) ${ }^{7}$. El afán de materializar la idea de la primacía romana supuso el despliegue de un amplio programa de reformas destinadas a imponer esa concepción eclesiológica: actividad conciliar, desarrollo de textos canónicos y jurídicos, legislación sinodal o, incluso, la celebración, en 1300, del primer jubileo romano. Una de las medidas más importantes, y que mayor eficacia tuvo en la consolidación de la centralización de la Iglesia y en la potenciación de la jerarquía romana, fue la reforma de la liturgia con la intención de generalizar a todo Occidente los usos característicos de la Curia papal ${ }^{8}$. Ello requirió de la elaboración y difusión de nuevos libros litúrgicos, entre los que el pontifical, destinado al uso exclusivo del obispo -o del pontífice como obispo de Roma-, jugó un papel fundamental: no solo se redactó una nueva versión, el llamado pontifical de la Curia romana del siglo XIII ${ }^{9}$, sino que, además, los manuscritos que la contenían fijaron una codificación visual e iconográfica que marcaría la liturgia episcopal en los siglos venideros. No en vano, la intensa actividad reformista y la pujanza económica de sus promotores atrajeron hacia Roma a numerosos artistas procedentes de diversas partes de Italia o, incluso, de fuera. Ellos fueron los responsables de la renovación en la apariencia de la ciudad ${ }^{10}$ y de una riquísima producción de manuscritos iluminados, que, como consecuencia de los avatares sufridos por la Corte papal y de diversas circunstancias, terminaron dispersándose y conservándose, en la actualidad, en múltiples bibliotecas.

En este sentido, se han estudiado los códices miniados de origen romano -muchos de ellos pontificales- que se conservan en diversas instituciones, principalmente italianas y francesas,

\footnotetext{
${ }^{2}$ No en vano, el inventario más antiguo que se conserva fue el que mandó realizar Bonifacio VIII (1294-1303) en 1295 con motivo de su ascenso al solio pontificio (Jullien De Pommerol, 2001). Véanse, asimismo, JuLLIEN DE Pommerol, Monfrin, 1991. Paravicini Bagliani, 2001.

3 Bilotta, 2003: 7. Monfrin, 1993.

${ }^{4}$ Para una introducción sobre la miniatura boloñesa, véase el ya clásico trabajo de Alessandro Conti (CoNTI, 1981). Sobre la región umbra, tras el estudio de Antonino Caleca (CALECA, 1969), se han sucedido numerosas aproximaciones, destacando la reciente síntesis de Marina Subbioni (SUBBioni, 2003).

5 Pace, 1984. Avril, Gousset, Rabel, 1984: 135-138.

${ }^{6}$ Entre los estudios más recientes, destacan Gousset, 2000. Bilotta, 2003; 2008; 2009.

7 Para una introducción sobre este contexto, véase Pennington, 1984.

8 Para una primera aproximación al respecto, véase VAN DIJK, HAZELDEN WALKER, 1960.

9 ANDRIEU, 1940. Véase también la reciente edición bilingüe latín-francés, precedida de un interesante estudio histórico, realizada por Goullet, LoBrichon, PALAzzo, 2004.

${ }^{10}$ Véase al respecto el clásico trabajo de KRAUTHEIMER, 1980: 203-228, especialmente.
} 
como la Biblioteca Apostólica Vaticana o la Bibliothèque Nationale de France ${ }^{11}$. Por el contrario, los fondos custodiados en los archivos y bibliotecas de la antigua Corona de Castilla apenas han merecido la atención de los investigadores $\mathrm{y}$, sin embargo, un análisis detallado muestra que no son pocos los manuscritos romanos realizados entre finales del siglo XIII y principios del XIV que se conservan en ellos. Si nos ceñimos a los pontificales, objeto de nuestra tesis ${ }^{12}$, hemos determinado que al menos seis ejemplares responden a la familia litúrgica de la Curia romana y fueron producidos en Roma en ese arco cronológico ${ }^{13}$.

De todos ellos destacan, por las similitudes de su iluminación y de su contenido, dos manuscritos: Sevilla, Biblioteca Capitular y Colombina, ms. 56-1-11'14 y Tui, Archivo Histórico Diocesano, ms. 3. Ambos pertenecen a la familia de los pontificales de la Curia romana del siglo XIII $\mathrm{y}$, concretamente, a la que Michel Andrieu calificó como versión mixta ${ }^{15}$, tal y como se comentará más adelante, pero ninguno de los dos figura entre los editados por el liturgista ${ }^{16}$. En el primer caso, no se había realizado ningún estudio exhaustivo de su contenido ni de su iluminación, con lo que únicamente se le había atribuido un genérico origen italiano en el siglo XIV, sin mayores precisiones, en las breves noticias catalográficas en que aparece mencionado ${ }^{17}$. El segundo ejemplar ha sido objeto de mayor atención historiográfica pero siempre se le han asignado un origen y una cronología, a nuestro juicio, erróneos: Cataluña en el siglo XIV ${ }^{18}$, Bolonia en la primera mitad del XIV ${ }^{19}$ e Italia central en el XV $X^{20}$.

El objetivo de este artículo es, por tanto, demostrar que ambos pontificales fueron realizados en Roma a finales del siglo XIII y que se insertan plenamente en el contexto de producción de manuscritos confeccionados a instancias de la Curia papal en ese periodo. Para ello, ante la casi total ausencia de referencias documentales precisas sobre su origen y sus trayectorias posteriores, hemos de proceder analizando en detalle el estilo e iconografía de sus miniaturas, y también su contenido y su materialidad, y poniendo todo ello en relación con otros ejemplares que la historiografía ha atribuido a los talleres romanos de esta época.

El pontifical de Sevilla ha sufrido una pérdida notable de soporte ${ }^{21}$ : conserva actualmente 235 folios, de $290 \times 197 \mathrm{~mm}$, con foliación romana posterior en tinta sepia y distribuidos en veinticuatro quiniones, varios de ellos incompletos ${ }^{22}$. Presenta reclamos horizontales, en rojo y negro,

1 Véase la nota 6.

12 Titulada "Liturgia y Poder en la Edad Media. Estudio de los pontificales en el antiguo Reino de León y Castilla", inscrita en la Universidade de Santiago de Compostela y realizada con el apoyo del Programa de Formación de Profesorado Universitario del Ministerio de Educación.

${ }^{13}$ Se trata de los siguientes manuscritos: Sevilla, Biblioteca Capitular y Colombina, ms. 56-1-11; Toledo, Biblioteca Capitular, mss. 38.28, 56.20, 60.1 y 60.2, y Tui, Archivo Histórico Diocesano, ms. 3.

14 Olim: Vitr. BB 148-19 y 81-6-11.

15 ANDRIEU, 1940: 229-233.

16 ANDRIEU, 1940: 3-4.

17 Janini, 1977: 278, n. ${ }^{\circ} 338$. Álvarez Márquez, 1999: 102-103. Kay, 2007: n. ${ }^{\circ} 909$.

18 Domínguez Bordona, 1933: II, n. ${ }^{\circ} 2162$.

19 Sicart Giménez, 1981: 137-148. Manso Porto, Yzquierdo Perrín, 1993: 496-499. Yarza Luaces, 2006: 32 33. LÓPEZ-MAYÁN, 2008.

${ }^{20} \mathrm{KAY}, 2007: \mathrm{n} .^{\circ} 1025$.

${ }^{21}$ Faltan los ff. 2-9, 11-21, 34-39, 53-60, 74-89, 111, 126-137, 142, 163-170, 187-190, 196-203, 206-213, 226-232, 236 y ss.

${ }^{22}$ La estructura del volumen es como sigue (entre corchetes se indican los folios perdidos y su ubicación al principio o al final del cuaderno): $1^{2[+8]}(1,10), 2^{10}(-), 3^{10}(22-31), 4^{4[+6]}(32,33,40,41), 5^{10}(42-51), 6^{2[+8]}(52,61), 7^{2[+8]}$ $(62,71), 8^{4[+6]}(72,73,80,81), 9^{10}(82-91), 10^{10}(92-101), 11^{9[+1]}(102-110), 12^{10}(112-121), 13^{10}(122-131), 14^{8[+2]}$ $(132-141), 15^{[1+] 9}(143-151), 16^{10}(152-161), 17^{2[+8]}(162,171), 18^{10}(172-182), 19^{8[+4]}(183,184,185,186,191,192$, $193,194), 20^{2[+8]}(195,204), 21^{2[+8]}(205,214), 22^{10}(215-224), 23^{2[+8]}(225,234), 24^{1+\text { pestaña }}(235)$.

Arch. esp. arte, LXXXv, 339, JULIO-SEPTIEMBRE 2012, 213-232, ISSN: 0004-0428 

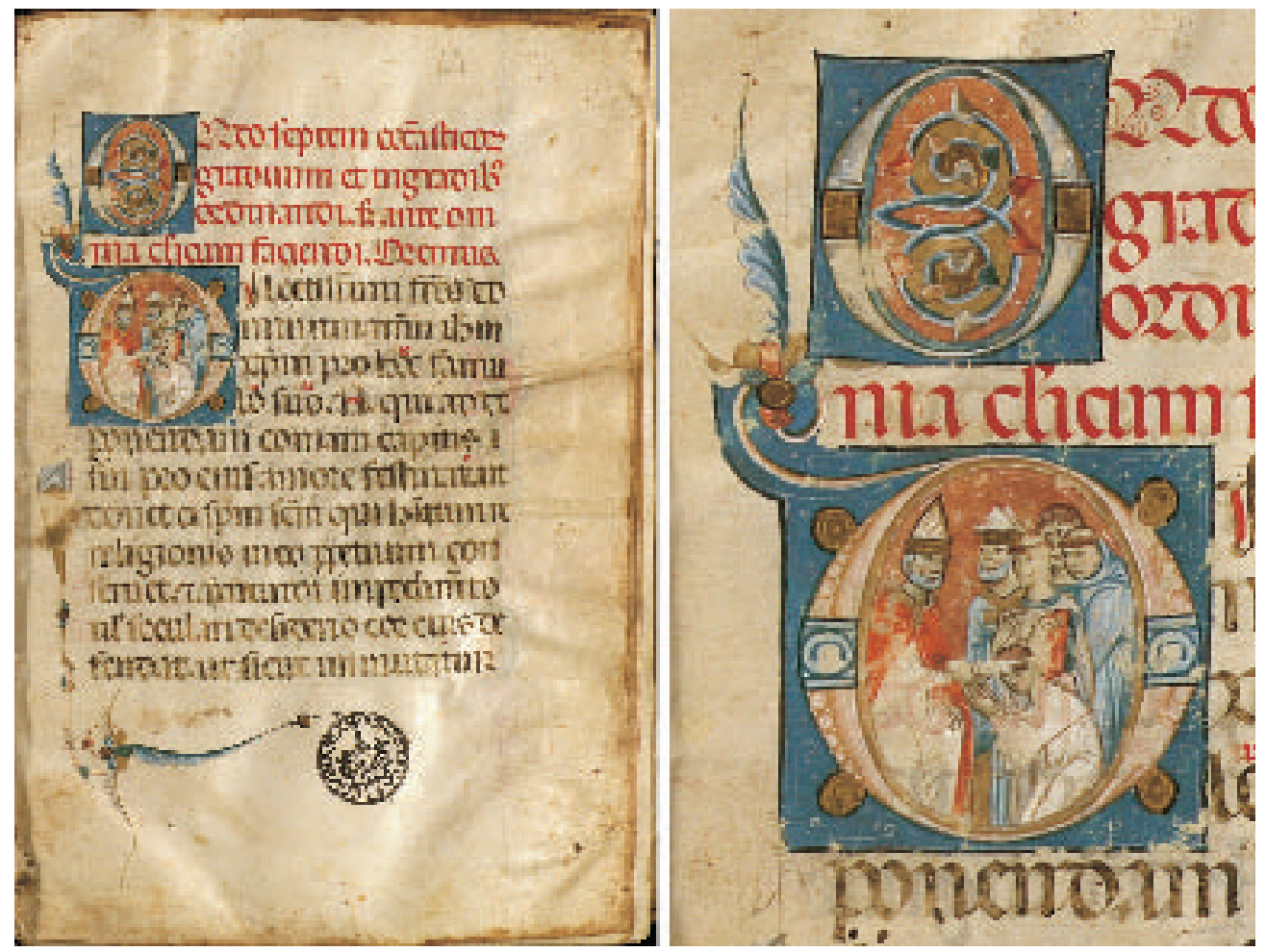

Fig. 1. Tonsura. Pontifical (ca. 1290-99). Sevilla, BCC, ms. 56-1-11, f. 1. (C) Biblioteca Capitular y Colombina.
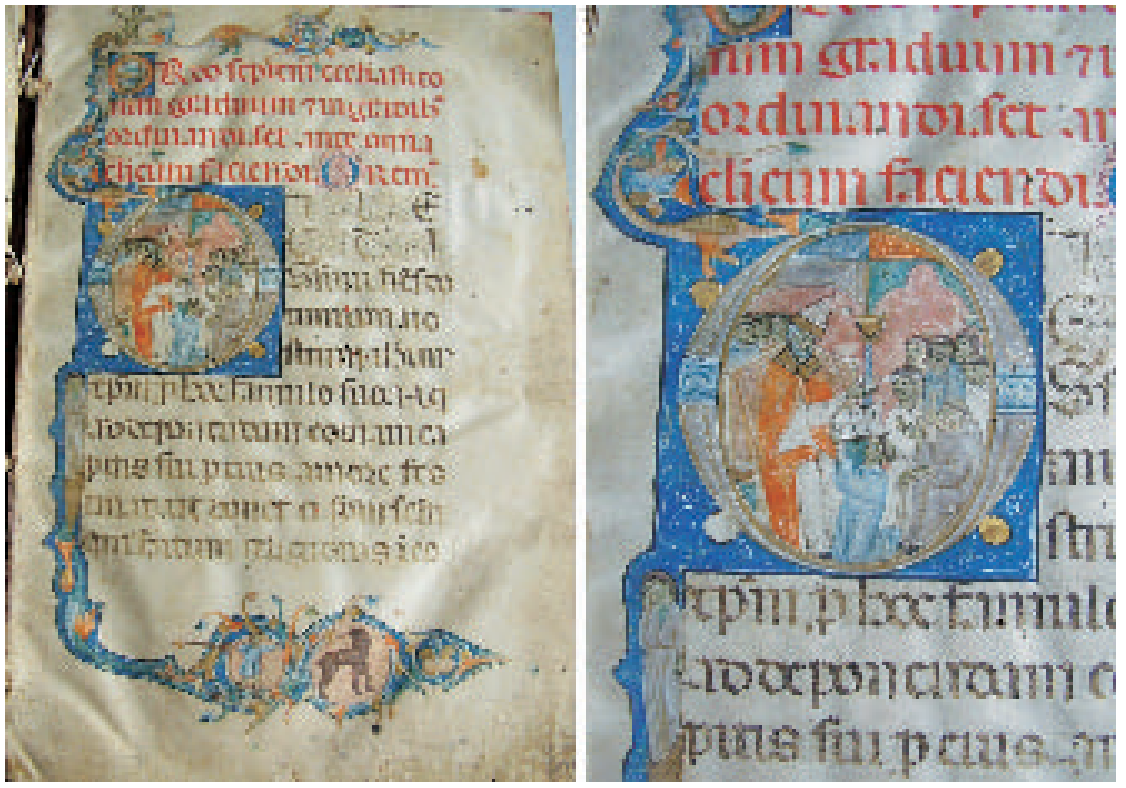

Fig. 2. Tonsura. Pontifical (ca. 1290-99). Tui, AHD, ms. 3, f. 1. (C) Archivo Histórico Diocesano de Tui. 


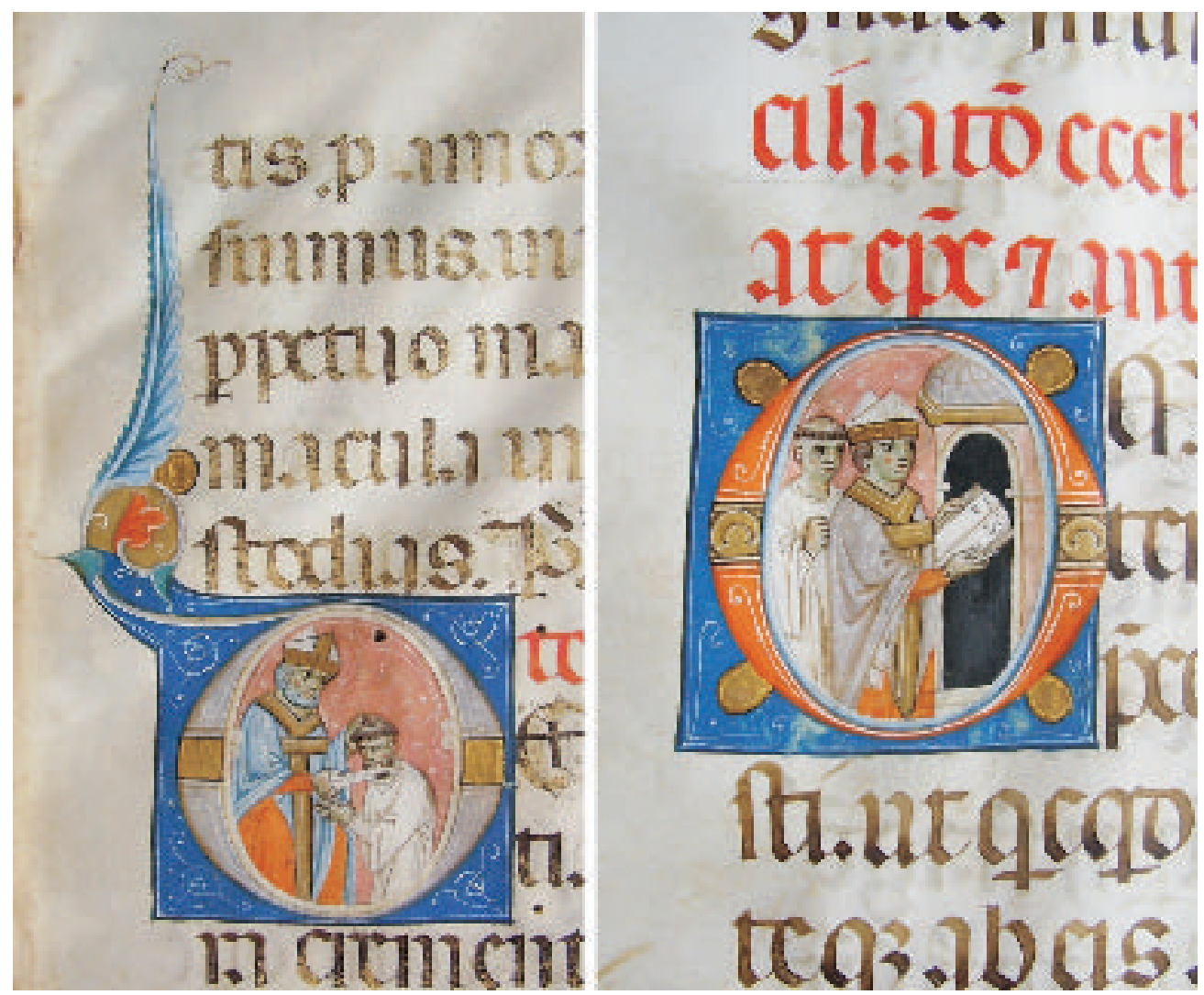

Fig. 3. Afeitado de la barba y reconciliación de la iglesia violada. Pontifical de Tui, ff. 3 y 153v.

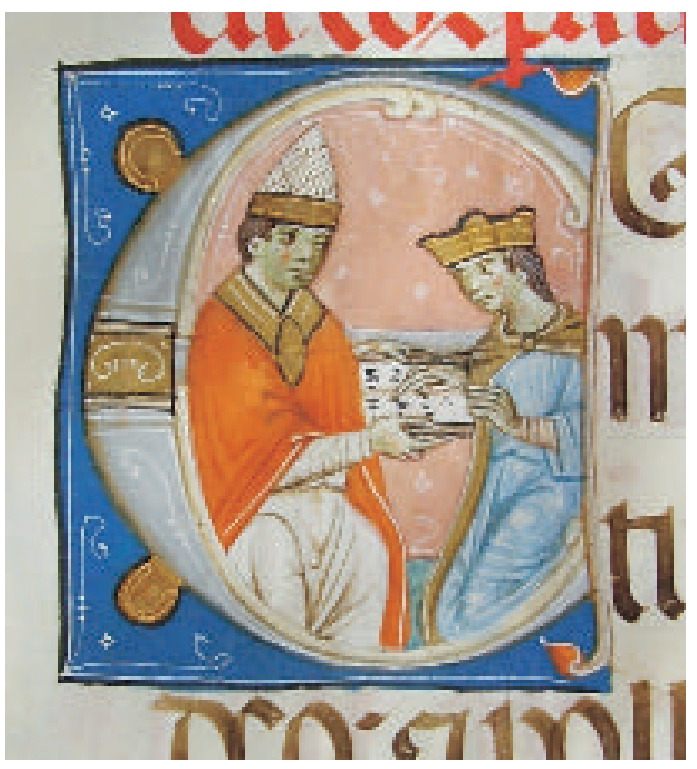

Fig. 4. Juramento del emperador. Pontifical de Tui, f. 60.

Arch. esp. arte, LXXXV, 339, JULIO-SEPTIEMBRE 2012, 213-232, ISSN: 0004-0428 
inscritos en recuadros geométricos y, en algunos casos, en figuras animales ${ }^{23}$, y está escrito en gótica libraria italiana, a una columna de $178 \times 126 \mathrm{~mm}$, con quince líneas pautadas a mina de plomo sin pinchazos visibles. Contiene notación musical cuadrada sobre tetragrama y posee una encuadernación del siglo XVII en pergamino con correíllas y sin decoración.

El pontifical de Tui tampoco está completo pues hay una laguna de texto entre los folios 188 y 189 , correspondiendo con el final del ordo XLII, el XLIII y el principio del XLIV. Contiene 270 folios, de $325 \times 218 \mathrm{~mm}$, con foliación reciente a lápiz y estructurados en veintiséis cuadernos, mayoritariamente quiniones ${ }^{24}$. En este caso, los reclamos están parcialmente eliminados por el desvirado posterior del volumen. También está escrito en gótica libraria italiana, a una columna de $202 \times 140 \mathrm{~mm}$, con catorce líneas pautadas a mina de plomo con pinchazos visibles solo en el último cuaderno. Contiene notación musical cuadrada sobre tetragrama y presenta una encuadernación de principios del siglo XIX realizada en piel castaña sobre madera con decoración dorada de cadeneta y pequeñas granadas en las esquinas.

La ornamentación principal de ambos volúmenes está constituida por iniciales historiadas, una en el ejemplar sevillano ${ }^{25}$ (fig. 1) y veinte en el tudense ${ }^{26}$ (figs. 2-5), que representan al obispo $-\mathrm{y}$, en ocasiones, al papa- desempeñando el ritual contenido en el texto al que acompañan, tal y como es habitual en los pontificales ${ }^{27}$. A ellas se añaden sendas iniciales iluminadas de modo similar a las historiadas pero solo con motivos vegetales (figs. 1-2) y una decoración secundaria integrada por iniciales de filigrana en rojo y azul de dos tamaños: 2 UR, con desarrollo bien de antenas caligráficas que se curvan y rematan en pequeñas espirales, bien de festones geométricos que ocupan el alto de la caja de escritura; y 1 UR, sin prolongaciones (fig. 6). Finalmente, encontramos también mayúsculas negras con motivos caligráficos y toques en rojo, y cruces de bendición y calderones de filigrana, alternando el rojo y el azul.

Los rasgos de la decoración constituyen el principal indicador, aunque no el único, del lugar y fecha de realización de ambos manuscritos. Tanto la iluminación del primer folio del pontifical

${ }^{23}$ Un pez en los folios $51 \mathrm{v}, 61 \mathrm{v}, 71 \mathrm{v}$ y $81 \mathrm{v}$, y un dragón en el folio $91 \mathrm{v}$.

${ }^{24}$ La estructura del volumen es como sigue: $1^{10}(1-10), 2^{10+1}(11-21), 3^{12}(22-33), 4^{10}(34-43), 5^{10}(44-53), 6^{10}(54-$ $63), 7^{10}(64-73), 8^{10}(74-83), 9^{10}(84-93), 10^{10}(94-103) 11^{10}(104-113), 12^{10}(114-123), 13^{8}(124-131), 14^{10}(132-141)$, $15^{16}(142-157), 16^{10+1}(158-168), 17^{10}(169-178), 18^{10}(179-188), 19^{10}(189-198), 20^{10}(199-208), 21^{10}(209-218), 22^{10}$ (219-228), $23^{10}$ (229-238), $24^{10}$ (239-248), $25^{10}$ (249-258), 26 $6^{10+2}$ (259-270: quinión formado por cuatro bifolios insertados entre dos guardas en blanco del ms. original -ff. 259 y $270-+$ dos folios desprendidos, con pestaña).

${ }^{25}$ Representa al pontífice tonsurando a un clérigo en presencia de otros cuatro eclesiásticos. Está situada en el f. 1, en el inicio del ordo I, y se prolonga en tallos vegetales decorados con nudos y pequeñas hojas y rematados, en espiral, por alargados acantos.

${ }^{26}$ Las escenas representadas son las siguientes: pontífice tonsurando a un clérigo en presencia de otros siete eclesiásticos y se acompaña de una bordura que enmarca la página inicial y que contiene varias figurillas humanas, dragones que se funden con los motivos de tallos, hojas y acantos enroscados, y, en el margen inferior, un unicornio y un arquero que se dispone a cazarlo, albergados en sendos tondos (f. 1, ordo I); obispo cortando la barba a un clérigo (f. 3, II); -entregando dos llaves al ostiario (f. 4, V); -entregando un libro al lector (f. 5, VI); -entregando al exorcista el rollo con la liturgia de los exorcismos (f. 6, VII); - entregando una vela al acólito (f. 8, VIII); -entregando un cáliz al subdiácono (f. 11, IX); pontífice bendiciendo a un diácono, en presencia de otros dos eclesiásticos y en el interior de una iglesia (f. 13v, X); obispo bendiciendo al presbítero, que lee las Escrituras, acompañado de otro eclesiástico (f. 19v, $\mathrm{X}$ ); -sentado en su cátedra, de frente, bendiciendo y sosteniendo un libro cerrado con su mano izquierda (f. 29, XI); -bendiciendo al pueblo (f. 40, XI); pontífice tomando juramento de fidelidad al emperador (f. 60, XVB); obispo bendiciendo al abad (f. 77v, XVI); - bendiciendo a un monje (f. 80v, XVII); -bendiciendo las vestimentas monásticas (f. 83v, XVII); -bendiciendo a una viuda (f. 92v, XIX); -bendiciendo las vestimentas del altar en el interior de la iglesia (f. 141, XXIII); -reconciliando la iglesia violada, asistido por otro eclesiástico (f. 153v, XXVI); -bendiciendo a un niño sostenido en brazos por su madre (f. 173, XXXIV); pontífice bendiciendo los santos óleos del Jueves Santo (f. 180v, XLII).

27 Para una introducción a esta cuestión, véanse PALAZZO, 1999. RABEL, 2009. 


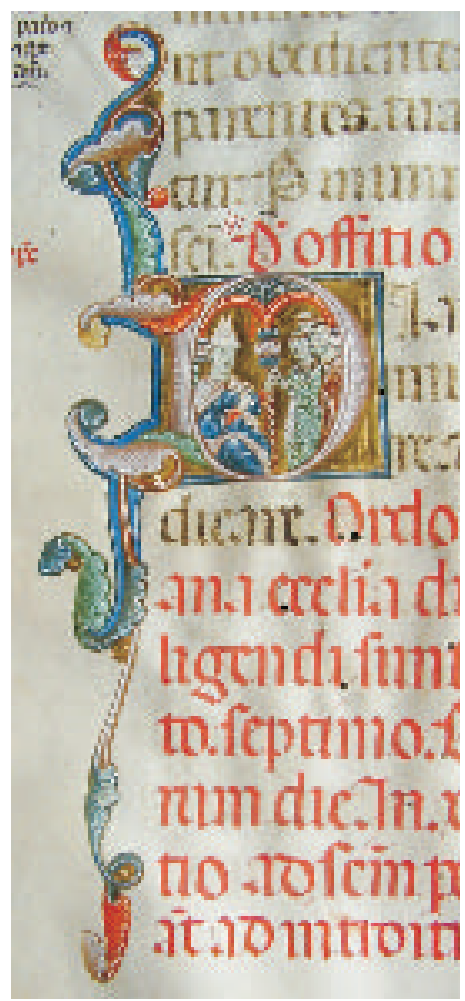

Fig. 5. Bendición del diácono. Pontifical de Tui, f. 13v.
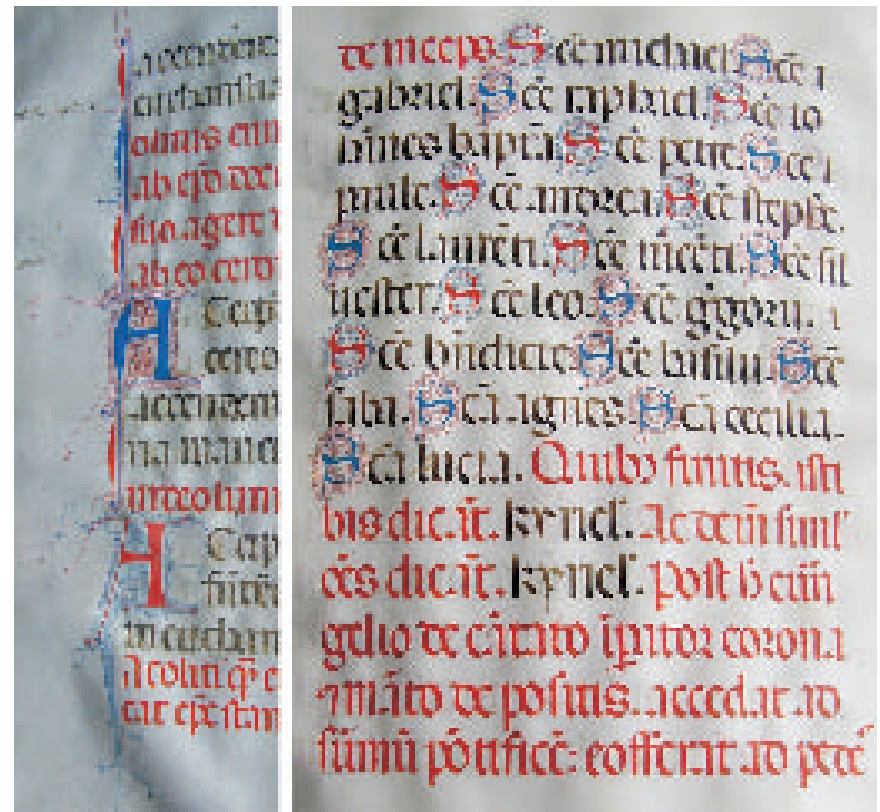

Fig. 6. Iniciales de filigrana. Pontifical de Tui, ff. $8 v$ y $17 v$. 
de Sevilla como la mano responsable de la mayoría de las iniciales del ejemplar tudense -todas, salvo la del f. 13v- acusan una fuerte influencia del estilo de la miniatura perusina debida al Maestro del Misal de Deruta y a los artistas que trabajaron en su órbita ${ }^{28}$. Activo en el último cuarto del siglo XIII en el contexto de eclosión artística que se produjo en Umbría paralelamente al desarrollo de las obras en la vecina basílica de Asís, dicho Maestro fue el responsable de iluminar, bajo la influencia directa del trabajo de Cimabue y de otros artistas, una gran cantidad de manuscritos ${ }^{29}$. Junto a éstos se han atribuido a su propia mano, o a la de algún discípulo, otros muchos códices, entre los que sobresale un grupo de ejemplares estilísticamente muy homogéneos y conservados en la Bibliothèque Nationale de France ${ }^{30}$. En conjunto, la decoración de todos estos manuscritos marcó las directrices de la miniatura umbra del final del Doscientos y, por su papel de intermediaria entre la cultura figurativa local y las novedades artísticas de Asís, condicionó la iluminación de manuscritos en esta región y en otras zonas de Italia durante las décadas siguientes.

Las analogías entre los pontificales de Sevilla y Tui y los manuscritos relacionados con el Maestro del Misal de Deruta son evidentes tanto en el tratamiento de las figuras como en la decoración. En el primer caso, los rostros están trazados a base de toques de sombreado en verde y con mejillas sonrosadas: la nariz es corta y recta, dibujada con un único trazo inferior, la comisura de los labios tiende a curvarse hacia abajo y solo el labio inferior está dibujado, frente a unos ojos grandes y expresivos provistos de bolsas casi triangulares, rasgos todos ellos característicos del citado Maestro ${ }^{31}$. Los cabellos y barbas están formados por sucesiones de líneas de color, grises o marrones, y se observan en los ropajes incipientes sombreados obtenidos mediante toques de color que les confieren una apariencia esquemática y geométrica ${ }^{32}$. Las escenas protagonizadas por los personajes así representados se caracterizan por la simplicidad compositiva, que focaliza la atención en los gestos rituales y que prescinde casi por completo de cualquier marco arquitectónico en beneficio de fondos planos de color rosa salpicados por detalles filiformes blancos.

En cuanto a la decoración, las iniciales tanto de los pontificales conservados en Castilla como de los manuscritos perusinos aludidos presentan unas mismas soluciones ornamentales ${ }^{33}$. Las letras se insertan en recuadros azul ultramar que se decoran con pequeños círculos o cuadrados blancos y con motivos filiformes, también blancos, que tienden a curvarse en las esquinas formando espirales de lo que parecen diminutos y esquemáticos tallos vegetales. Estos elementos filiformes aparecen igualmente en los cuerpos de las letras, que se ensamblan a través de motivos

28 Sobre la actividad del Maestro del Misal de Deruta, sobre los manuscritos que se le han atribuido y sobre su influencia, véanse Lunghi, 1992; 2004. Neri LusANNA, 1982. SubBioni, 2001.

29 Entre ellos, destacan el Misal de Deruta, al que debe su nombre (Deruta, Archivio e Pinacoteca Comunale, ms. [sine signatura]), un Gradual conservado en Perugia (Biblioteca Capitolare, ms. 16) y el más antiguo de los Antifonarios de San Domenico (Perugia, Biblioteca Augusta, ms. 2783) (SuBbioni, 2003: 12).

${ }^{30}$ De los nueve manuscritos que representan la miniatura umbra en los fondos de la BNF, dos son, sin duda, originarios de la propia Perugia; se trata de una Biblia (París, BNF, ms. lat. 41) y del De regimine christiano de Jacques de Viterbo (París, BNF, ms. lat. 4229, ff. 59-112) (AvRiL, Gousset, Rabel, 1984: n. ${ }^{\text {os }} 143$ y 149, respectivamente). Los demás pudieron, incluso, ser realizados fuera de esta ciudad por artífices perusinos o influidos por el estilo de Perugia (Avril, Gousset, RaBel, 1984: n. $^{\text {os }} 144,145,146,147,148,150$ y 151).

31 SubBioni, 2003: 11.

32 Compárese, por ejemplo, con la inicial que representa a san Pedro en la Biblia umbra de París, BNF, ms. lat. 41, f. 441 (Avril, Gousset, RABEL, 1984: lám. I), digitalizada en la web <http://images.bnf.fr/jsp/index.jsp> [Consulta: 11 de marzo de 2011]; o con la inicial de las santas mujeres del Gradual de Perugia, Biblioteca Capitolare, ms. 16, f. 98v, reproducida en CALECA, 1969: lám. 407.

33 Neri Lusanna, 1982: 181. Avril, Gousset, Rabel, 1984: 117-118. Compárese con las iniciales del Gradual de Perugia, Biblioteca Capitolare, ms. 16, f. 98v, reproducidas en CALECA, 1969: 405-420; o con las iniciales de la Biblia de París, BNF, ms. lat. 41, reproducidas en MARques, 1987: 170-171. 
geométricos, generalmente en oro, se decoran con pequeños círculos de color o con acantos muy esquemáticos y casi siempre están bordeados interiormente por una franja de color más claro. Es frecuente la presencia de glóbulos de oro en los bordes de las iniciales y generalmente éstas se prolongan en tallos vegetales que, salpicados por pequeñas hojas de perfil redondeado, se enroscan formando espirales que suelen compartir el mismo fondo azul ultramar que el resto de la letra; únicamente sobresalen del conjunto, prologándose por los márgenes de los folios, largos acantos con perfiles dentellados y gruesas nervaduras realizadas en el mismo color que las hojas pero con tonalidades diferentes. Incluso, se ha considerado como un elemento característico del aparato decorativo del Maestro de Deruta la presencia de borduras con forma de dragón, presentes en el primer folio del pontifical de Tui ${ }^{34}$.

La iluminación de todos estos manuscritos se caracteriza, además, por una gran intensidad cromática, con predominio de tonos vivos (rojos, naranjas, azules, verdes...), que solo el empleo del gris, el beige o el azul claro de las túnicas logra atemperar, aportando, en conjunto, una luminosidad típica de la miniatura umbra ${ }^{35}$.

Pese a todo, la intervención de una segunda mano en la decoración del pontifical de Tui -en la inicial del f. $13 \mathrm{v}$ - hace pensar que no se trata de un manuscrito realizado en Perugia (fig. 5). El esquema compositivo y ornamental de la letra es muy similar al que presentan las restantes iniciales del códice, pero sus rasgos denotan un artífice mucho más negligente. Se observa una mayor dificultad en la representación gestual y una mayor rigidez, fruto de la ausencia de sombreado en los ropajes y del recurso, en su lugar, a gruesas líneas blancas que dibujan los pliegues de las túnicas. La inicial misma se inserta en un recuadro dorado, caso insólito en el manuscrito, y la decoración vegetal con que se prolonga por el margen está mucho más desarrollada que en el resto de las letras del pontifical; sin embargo, los tallos y las hojas se alejan de las refinadas formas visibles en otras iniciales y se hacen mucho más carnosos y toscos, a lo que también contribuyen los marcados perfiles y nervaduras de unos acantos cuyos anchos extremos se curvan alejándose de los estilizados remates presentes en el resto del volumen.

Todo ello nos permite suponer que el responsable de esta inicial trató de imitar el estilo de Perugia del resto de las letras pero su menor habilidad hizo que el resultado fuera bastante diferente. Y esto nos remite a otros pontificales de la Curia romana iluminados de manera similar a los manuscritos umbros pero con ciertas variantes en el estilo; tal es el caso, por ejemplo, del ms. lat. 965 de la Bibliothèque Nationale de France ${ }^{36}$, decorado a finales del siglo XIII por cinco artistas, uno de los cuales, el principal, está muy próximo a dos ejemplares perusinos (Perugia, Biblioteca capitolare, ms. 15, y Perugia, Biblioteca Comunale Augusta, ms. 2792), mientras que los otros cuatro, mediocres imitadores, tienen tendencia a simplificar los motivos, algo que se ha interpretado como muestra de que fue ejecutado en Roma pero bajo una fuerte influencia de la miniatura umbra ${ }^{37}$.

El sincretismo estilístico es uno de los rasgos más característicos de la miniatura realizada en Roma en la medida en que su condición de centro de la cristiandad -con la capacidad económica y la influencia política que ello conllevaba- convirtió a esta ciudad en un foco de atracción, reinterpretación y difusión de las obras de artistas procedentes de diversos territorios, incluso fuera de Italia, que aportaron sus connotaciones estilísticas regionales ${ }^{38}$. En lo que se refiere a la miniatura umbra, su importancia en este proceso se hizo crucial en las últimas décadas del si-

\footnotetext{
34 TodINI, 1982: 162.

35 AVRIL, 1984: 47.

${ }^{36}$ Véase la digitalización de los ff. 1, 24 y 32 de este manuscrito en la web $<$ http://images.bnf.fr/jsp/index.jsp $>$ [Consulta: 11 de marzo de 2011].

37 Avril, Gousset, Rabel, 1984: n. ${ }^{\circ} 164$.

38 De Benedictis, Neri Lusanna, 1990: 9.
} 
glo XIII, coincidiendo con la efervescencia artística en torno a la basílica de Asís: durante los pontificados de Martín IV (1281-1285) y de Honorio IV (1285-1287) la Curia papal residió en Perugia $^{39}$ y en 1288 ascendió a la cátedra de san Pedro Nicolás IV (1288-1292), franciscano de origen umbro que, además de reforzar la influencia de esta Orden en la Corte pontificia, impulsó las relaciones entre Roma y esta región ${ }^{40}$. En este contexto se iluminaron gran cantidad de manuscritos, muchos de ellos litúrgicos, con los que los pontificales de Tui y Sevilla presentan estrechas relaciones estilísticas. Además del ya mencionado ms. lat. 965 y entre otros ejemplares ${ }^{41}$, cabe citar unas Chroniques d'Outremer de Guillaume de Tyr (París, BNF, ms. français 9082 ${ }^{42}$ ), dotadas de un excepcional colofón que data la realización del manuscrito en Roma en 1295 y permite tener, así, una referencia espacial y temporal para este conjunto de códices y, por tanto, también para los pontificales de Castilla ${ }^{43}$.

La nómina de manuscritos iluminados en Roma en las últimas décadas del Doscientos bajo la influencia de la miniatura umbra es mucho más amplia ${ }^{44}$ e incluye también una serie de códices en los que los rasgos umbro-romanos se combinan con elementos procedentes de otras regiones italianas, notoriamente Bolonia ${ }^{45}$. En estos casos, se observa una mayor distancia estilística con respecto a los pontificales de Tui y Sevilla, donde los elementos de origen boloñés son prácticamente inexistentes ${ }^{46}$. Su importancia reside, entonces, en que, entre estos códices, se cuentan varios pontificales de la Curia romana (especialmente, París, BNF, ms. lat. $960^{47}$, y Ciudad del Vaticano, BAV, ms. Vat. lat. $1155^{48}$ ) con los que los ejemplares españoles se pueden relacionar desde el punto de vista iconográfico. La iconografía es, en efecto, el segundo indicador que, junto a los rasgos estilísticos, nos permite situar la realización de los pontificales de Castilla en la Curia papal durante las últimas décadas del siglo XIII.

Al igual que ocurre en los manuscritos lat. 960 y Vat. lat. 1155, en las iniciales historiadas contenidas en los ejemplares de Tui y Sevilla subyace la voluntad de representar un ciclo de imágenes que, en primer lugar, mostrase visualmente la importancia de la liturgia compendiada en el propio libro pontifical ${ }^{49}$. Por ello las escenas se resuelven con gran sencillez compositiva,

39 Gousset, 2000: 109. Véanse, asimismo, Paravicini Bagliani, 1988: 155-278; 1995: 31 y ss.

40 Ciardi Dupré dal Poggetto, 2005: 202-203.

${ }^{41}$ Es el caso de un grupo de cinco misales (Ciudad del Vaticano, Biblioteca Apostolica Vaticana, mss. Arch. S. Pietro B71 y E7 y Santa Maria Maggiore 91 y 92) (De Benedictis, Neri Lusanna, 1990: 10 y figs. 1, 2, 5, 9, 10, 11, 15 y 17) y de otro pontifical de la Curia (Ciudad del Vaticano, Biblioteca Apostólica Vaticana, ms. Vat. lat. 4747) (Gousset, 2000: 185-186).

42 Véanse algunas de las numerosas iniciales historiadas de este manuscrito -especialmente, la contenida en el f. 241- en la web <http://images.bnf.fr/jsp/index.jsp> [Consulta: 11 de marzo de 2011].

43 Avril, Gousset, RABEL, 1984: 137-138. Sobre este manuscrito, véanse también Folda, 1973: 95; 1976: 94 , $133-138,140,146,156,168$ y 200-20.

${ }^{44}$ Y continúa incrementándose; recientemente, François Avril ha identificado otros dos manuscritos realizados en este contexto artístico romano: Nuremberg, Stadtbibliothek, ms. Cent.III.91, y Frankfurt, Städel Museum, Inv. 13935. Queremos dejar constancia de nuestro profundo agradecimiento a F. Avril no solo por habernos facilitado esta información sino también por su inestimable y desinteresada ayuda en la resolución de algunas de las cuestiones planteadas por los pontificales que aquí analizamos.

45 De Benedictis, Neri Lusanna, 1990: 12-13.

46 Solo puede relacionarse con la presencia de influjos boloñeses en los manuscritos romanos de este grupo el pequeño rostro humano que emerge de la decoración vegetal en la inicial del f. 13v del pontifical de Tui, similar a la presente en el misal Ciudad del Vaticano, BAV, ms. Chigi C.VII 210, f. 7 (De Benedictis, Neri Lusanna, 1990: fig. 29).

47 Sobre este manuscrito véanse, principalmente, Avril, Gousset, Rabel, 1984: n. ${ }^{\circ}$ 163. Palazzo, 1999: 149, 151, $153,200,205,210,212,220,223,232,235,266,269,272,276,322,324,328,355$. Véase, asimismo, la digitalización de este manuscrito en la web <http://images.bnf.fr/jsp/index.jsp $>$ [Consulta: 11 de marzo de 2011].

48 Sobre este manuscrito véase BILOTTA, 2003: 40-41.

49 BilotTA, 2009: 65. 
poniendo el acento únicamente en el gesto ritual que relaciona -jerárquicamente- al obispo con los demás miembros de su Iglesia. Y por ello, asimismo, las imágenes poseen un claro carácter ilustrativo, siguiendo estrictamente las descripciones contenidas en las rúbricas correspondientes. Esto se traduce en una estrecha tipificación del ciclo iconográfico de los pontificales realizados en la Curia y explica las coincidencias entre estos manuscritos y los ejemplares de Castilla: catorce de las veinte iniciales del pontifical de Tui son iconográficamente idénticas -y tienen la misma situación dentro del texto- a dieciséis de las cuarenta y nueve iniciales que presenta el ms. lat. 960. Frente a ello, en el caso de otros pontificales, como el de Autun ${ }^{50}$, realizado en estilo boloñés, hacia 1300 y con la versión larga del texto, solo son ocho las iniciales en las que la iconografía del pontifical de Tui es similar (tabla I).

Tabla I. Comparación de la decoración de cuatro pontificales de la Curia romana ${ }^{51}$

\begin{tabular}{|c|c|c|c|c|c|c|c|c|c|}
\hline \multirow{2}{*}{$\begin{array}{c}\text { Ordo } \\
\mathrm{I}\end{array}$} & \multirow{2}{*}{$\begin{array}{c}\text { Motivo } \\
\text { Tonsura }\end{array}$} & \multicolumn{2}{|c|}{ Pontifical Sevilla } & \multicolumn{2}{|c|}{ Pontifical Tui } & \multicolumn{2}{|c|}{ Pontifical Autun } & \multicolumn{2}{|c|}{ Pontifical BNF lat. 960} \\
\hline & & 1 & $\mathrm{D}$ (ilectissimi) & 1 & $\mathrm{D}$ (ilectissimi) & 1 & $\mathrm{O}$ (remus) & $1 \mathrm{v}$ & $\mathrm{O}$ (remus) \\
\hline II & Barba & - & & 3 & $\mathrm{D}$ (eus) & & & & \\
\hline $\mathrm{V}$ & Hostiario & - & & 4 & $\mathrm{H}($ ostiarium $)$ & $2 \mathrm{v}$ & $\mathrm{O}($ stiarium $)$ & 4 & $\mathrm{H}($ ostiarium $)$ \\
\hline VI & Lector & - & & 5 & $\mathrm{~L}($ ectorem$)$ & 3 & $\mathrm{~L}($ ectorem) & 5 & $\mathrm{~L}($ ectorem$)$ \\
\hline VII & Exorcista & - & & 7 & $\mathrm{E}$ (xorciste) & 4 & E(xorcistam) & $6 \mathrm{v}$ & E(xorcistam) \\
\hline VIII & Acólito & - & & 8 & $\mathrm{~A}($ colitum $)$ & $4 v$ & $\mathrm{~A}($ colitum $)$ & 8 & $\mathrm{~A}($ colitum $)$ \\
\hline IX & Subdiácono & - & & 11 & S(ubdiaconum) & $5 \mathrm{v}$ & S(ubdiaconum) & 10 & S(ubdiaconum) \\
\hline $\mathrm{X}$ & $\begin{array}{c}\text { Elección } \\
\text { diácono }\end{array}$ & - & & - & & 7 & $\mathrm{O}(\mathrm{rdo})$ & - & \\
\hline $\mathrm{X}, 1$ & $\begin{array}{c}\text { Ordenación } \\
\text { diácono }\end{array}$ & - & & $13 \mathrm{v}$ & $\mathrm{D}$ (iaconum) & - & & $12 \mathrm{v}$ & $\mathrm{D}$ (iaconum) \\
\hline $\mathrm{X}, 12$ & $\begin{array}{l}\text { Ordenación } \\
\text { sacerdote }\end{array}$ & - & & - & & $10 \mathrm{v}$ & $\mathrm{O}$ (rdinatio) & - & \\
\hline $\mathrm{X}, 16$ & & - & & $19 \mathrm{v}$ & S(acerdotem) & - & & $17 \mathrm{v}$ & S(acerdotem) \\
\hline $\mathrm{XI}$ & Ordo del obispo & - & & 29 & I(ncipit ordo) & 19 & I(ncipit ordo) & 25 & I(ncipit ordo) \\
\hline XI, 13 & & - & & - & & - & & $29 v$ & $\mathrm{~A}$ (ntiqua) \\
\hline XI, 19 & & - & & 40 & E(piscopum) & - & & 34 & \begin{tabular}{|l|} 
A por \\
E(piscopum)
\end{tabular} \\
\hline XIIIA & Ordo del papa & - & & - & & 36bis & I(ncipit ordo) & 49 & I(ncipit ordo) \\
\hline XIII, 2 & & - & & - & & - & & $52 \mathrm{v}$ & A(desto) \\
\hline XIII, 3 & & - & & - & & - & & $52 \mathrm{v}$ & $\mathrm{P}$ (ropiciare) \\
\hline $\mathrm{XIV}, 2$ & & - & & - & & - & & $54 \mathrm{v}$ & D(eus qui) \\
\hline XVB & $\begin{array}{c}\text { Ordo del } \\
\text { emperador }\end{array}$ & - & & - & & $44 v$ & C(um rex) & $55 \mathrm{v}$ & C(um rex) \\
\hline $\mathrm{XV}, 6$ & $\begin{array}{l}\text { Sermón del } \\
\text { emperador }\end{array}$ & - & & 60 & $\mathrm{E}$ (go enim) & - & & - & \\
\hline $\mathrm{XV}, 20$ & $\begin{array}{l}\text { Misar por el } \\
\text { emperador }\end{array}$ & - & & - & & - & & $61 \mathrm{v}$ & \begin{tabular}{|l|}
$\mathrm{D}($ eus \\
regnorum $)$
\end{tabular} \\
\hline $\mathrm{XV}, 44$ & $\begin{array}{l}\text { Coronación } \\
\text { reina }\end{array}$ & - & & - & & 54 & S(i vero) & $69 \mathrm{v}$ & S(i vero) \\
\hline
\end{tabular}

50 DAmongeot, AvriL, 2011.

51 Tanto la estructura de la tabla como la información relativa al pontifical de Autun y al ejemplar de París, BNF, ms. lat. 960, están tomadas de DAMONGEOT, AvRIL, 2011: 70-71, donde se define la decoración de otros tres pontificales más de la misma época. Nuestra intención es la de integrar los ejemplares de Tui y Sevilla en este cuadro para comprender las relaciones iconográficas que existen entre éstos y otros manuscritos contemporáneos. 
Tabla I. Comparación de la decoración de cuatro pontificales de la Curia romana (Cont.)

\begin{tabular}{|c|c|c|c|c|c|c|c|c|c|}
\hline \multirow{2}{*}{$\begin{array}{l}\text { Ordo } \\
\text { XVI }\end{array}$} & \multirow{2}{*}{$\begin{array}{c}\text { Motivo } \\
\begin{array}{c}\text { Bendición del } \\
\text { abad }\end{array}\end{array}$} & \multicolumn{2}{|c|}{ Pontifical Sevilla } & \multicolumn{2}{|c|}{ Pontifical Tui } & \multicolumn{2}{|c|}{ Pontifical Autun } & \multicolumn{2}{|c|}{ Pontifical BNF lat. 960} \\
\hline & & - & & - & & 61 & $\begin{array}{l}\mathrm{I}(\mathrm{n} \\
\text { benedictione })\end{array}$ & - & \\
\hline XVI, 16 & & - & & $77 \mathrm{v}$ & Q(uesumus) & - & & 74 & $\mathrm{Q}$ (uesumus) \\
\hline XVII & $\begin{array}{l}\text { Toma de } \\
\text { hábitos }\end{array}$ & - & & $80 \mathrm{v}$ & $\mathrm{A}($ desto $)$ & $61 \mathrm{bis}$ & $\mathrm{O}(\mathrm{rdo})$ & $77 \mathrm{v}$ & $\mathrm{A}($ desto $)$ \\
\hline XVII, 6 & $\begin{array}{l}\text { Bendición } \\
\text { hábitos }\end{array}$ & - & & $83 \mathrm{v}$ & $\mathrm{D}$ (omine) & - & & - & \\
\hline XVIII & $\begin{array}{l}\text { Bendición } \\
\text { virgen }\end{array}$ & - & & - & & 63 & $\mathrm{O}(\mathrm{rdo})$ & - & \\
\hline XVIII, 3 & $\begin{array}{l}\text { Bendición } \\
\text { hábitos }\end{array}$ & - & & - & & - & & 81 & $\mathrm{D}$ (eus) \\
\hline XIX & Bendición viuda & - & & $92 \mathrm{v}$ & $\mathrm{C}$ (onsolare) & 73 & $\mathrm{C}$ (onsolare) & $88 \mathrm{v}$ & $\mathrm{C}$ (onsolare) \\
\hline $\mathrm{XX}$ & $\begin{array}{c}\text { Alforjas y } \\
\text { bastón }\end{array}$ & - & & - & & 74 & $\mathrm{D}$ (omine) & - & \\
\hline $\mathrm{XX}, 5$ & $\begin{array}{l}\text { Cruz del } \\
\text { peregrino }\end{array}$ & - & & - & & 75 & S(uper) & - & \\
\hline XXI & Primera piedra & - & & - & & $75 v$ & $\mathrm{~B}$ (enedic) & - & \\
\hline XXIII & Iglesia y altar & - & & - & & $75 v$ & $\mathrm{O}(\mathrm{rdo})$ & - & \\
\hline $\begin{array}{c}\text { XXIII, } \\
27\end{array}$ & Exorcismo sal & - & & - & & - & & 93 & $\mathrm{E}($ xorcizo) \\
\hline $\begin{array}{c}\text { XXIII, } \\
31\end{array}$ & $\begin{array}{l}\text { Bendición } \\
\text { cenizas }\end{array}$ & - & & - & & - & & 105 & $\mathrm{H}$ (omnipotens) \\
\hline $\begin{array}{c}\text { XXIII, } \\
69 \\
\end{array}$ & $\begin{array}{c}\text { Consagración } \\
\text { altar }\end{array}$ & - & & - & & - & & 125 & Q(uesumus) \\
\hline $\begin{array}{c}\text { XXIII, } \\
75 \\
\end{array}$ & $\begin{array}{c}\begin{array}{c}\text { Vestimentas del } \\
\text { altar }\end{array} \\
\end{array}$ & - & & 141 & $\mathrm{D}$ (omine) & - & & $128 \mathrm{v}$ & $\mathrm{D}$ (omine) \\
\hline XXIV & Cementerio & - & & - & & $109 \mathrm{v}$ & $\mathrm{D}$ (omine) & 135 & $\mathrm{D}$ (omine) \\
\hline XXV & Campana & - & & - & & 110 & $\mathrm{O}(\mathrm{rdo})$ & $135 \mathrm{v}$ & $\mathrm{O}(\mathrm{rdo})$ \\
\hline XXVI & $\begin{array}{l}\text { Reconciliación } \\
\text { iglesia }\end{array}$ & - & & $153 \mathrm{v}$ & $\mathrm{O}$ (mnipotens) & $113 \mathrm{v}$ & $\mathrm{R}$ (econciliatio) & 140 & $\mathrm{O}$ (mnipotens) \\
\hline XXVII & $\begin{array}{c}\text { Piedra del altar } \\
\text { portátil }\end{array}$ & - & & - & & $119 \mathrm{v}$ & $\mathrm{D}$ (eum) & 145 & $\mathrm{D}$ (eum) \\
\hline XXVII, 2 & - & & - & & & $145 \mathrm{v}$ & E(xaudi) & & \\
\hline XXVIII & Patena & - & & - & & 124 & $\mathrm{O}$ (remus) & - & \\
\hline XXIX & Cáliz & - & & - & & $124 \mathrm{v}$ & $\mathrm{O}$ (remus) & - & \\
\hline XXX & Corporales & - & & - & & 125 & $\mathrm{C}($ lementissime $)$ & 151 & $\mathrm{C}$ (lementissime) \\
\hline XXXI & Cruz nueva & - & & - & & $125 \mathrm{v}$ & $\mathrm{B}$ (enedic) & $151 \mathrm{v}$ & $\mathrm{B}$ (enedic) \\
\hline XXXII & Incienso & - & & - & & $129 \mathrm{v}$ & $\mathrm{D}$ (omine) & $154 \mathrm{v}$ & $\mathrm{D}$ (omine) \\
\hline XXXIII & Casulla & - & & - & & $129 \mathrm{v}$ & $\mathrm{O}$ (mnipotens) & 155 & $\mathrm{O}$ (mnipotens) \\
\hline XXXIV & Niños & - & & 173 & $\mathrm{O}$ (mnipotens) & 130 & $\mathrm{O}(\mathrm{rdo})$ & - & \\
\hline XXXV & Pan & - & & - & & 131 & $\mathrm{D}$ (omine) & $157 \mathrm{v}$ & $\mathrm{D}$ (omine) \\
\hline XXXVI & Cordero & - & & - & & $131 \mathrm{v}$ & $\mathrm{D}$ (eus) & 158 & $\mathrm{D}$ (eus) \\
\hline XXXVII & Leche y miel & - & & - & & 132 & $\mathrm{~B}$ (enedic) & 159 & $\mathrm{~B}$ (enedic) \\
\hline XXXVIII & Queso y huevos & - & & - & & 132 & C(reator) & - & \\
\hline XXXIX & Frutos nuevos & - & & - & & 132 & $\mathrm{~B}$ (enedic) & - & \\
\hline $\mathrm{XL}$ & Uvas & - & & - & & $132 \mathrm{v}$ & B(enedic) & - & \\
\hline XLI & Nueva casa & - & & - & & $132 \mathrm{v}$ & $\mathrm{D}$ (eum) & - & \\
\hline XLII & Jueves santo & - & & - & & 132bis & I(ncipit ordo) & - & \\
\hline XLII, 13 & Santos óleos & - & & $180 \mathrm{v}$ & E(xorciço) & - & & 164 & $\mathrm{E}$ (mitte) \\
\hline
\end{tabular}


Tabla I. Comparación de la decoración de cuatro pontificales de la Curia romana (Cont.)

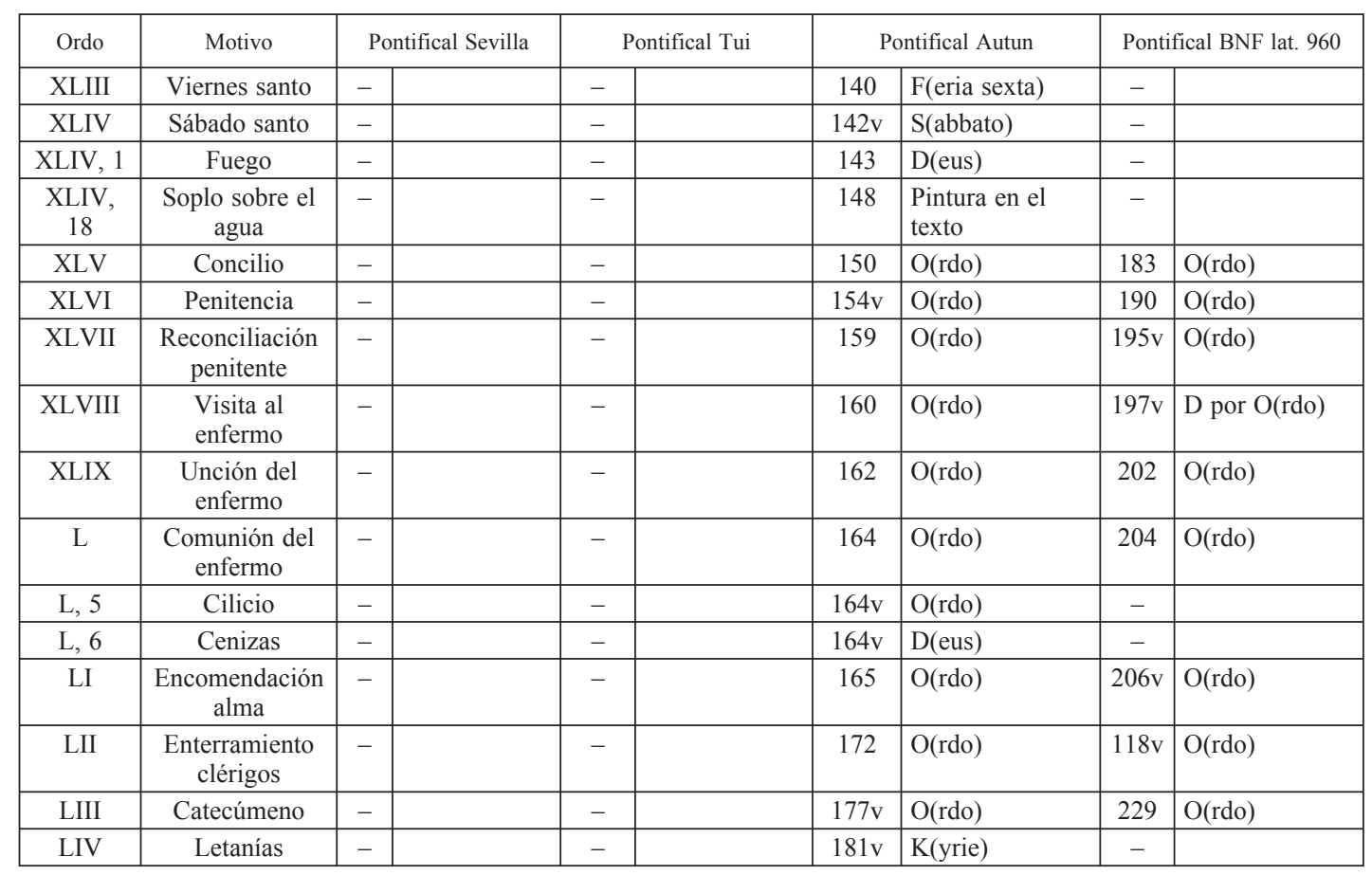

Solo en un caso texto e imagen difieren. En la inicial contenida en el f. 60 del manuscrito de Tui (fig. 4), al comienzo del ordo XV, ordo ad benedicendum seu coronandum imperatorem, es el pontífice quien sostiene el libro sobre el que el emperador debe pronunciar el juramento de fidelidad a la Iglesia y, sin embargo, en la rúbrica que la precede se indica que Quo demum surgente, rex ipse a parte dextera et prior diaconorum a parte sinistra deducant eum usque ad ecclesiam sancte Marie in Turribus, ubi ante altare, subdiacono evangelii textum tenente, rex super eum corporaliter prestet huiusmodi iuramentum (f. 59v). Ello se relaciona con el segundo valor que subyace en la iconografía de estos pontificales: el ideológico. Tanto en la inicial del ejemplar de Sevilla como en cuatro de las letras del pontifical de Tui ${ }^{52}$, es el papa, y no el obispo, quien preside la iconografía ritual (figs. 1, 2, 4, 5). La presencia del pontífice, también habitual en los mss. lat. 960 y Vat. lat. 1155, debe ponerse en relación con el contexto de renovación religiosa y refuerzo de la autoridad papal que se produjo, tal y como se comentó anteriormente, en el siglo XIII y una de cuyas manifestaciones fue, precisamente, la reforma de la liturgia y la elaboración del nuevo pontifical de la Curia romana. Dicho de otro modo, la ilustración del pontífice convertía visualmente en instrumentos de ostentación y propaganda del poder del papa y su Curia a unos manuscritos que textualmente ya lo eran desde su misma concepción bajo la iniciativa de Inocencio III (1198-1216) ${ }^{53}$.

Desde este punto de vista, los mss. lat. 960 y Vat. lat. 1155 han sido considerados por la historiografía como los ejemplos más antiguos que se conservan del ciclo iconográfico propio de

52 Véase nota 26.

53 Goullet, Lobrichon, Palazzo, 2004: 20. 
los pontificales romanos, que alcanzaría una enorme difusión en los siglos siguientes ${ }^{54}$. Creemos que la cercanía iconográfica de los ejemplares de Tui y Sevilla, unida a su datación también a finales del siglo XIII en función de la similitud estilística con otros manuscritos de esa época, permite incluir los dos pontificales de Castilla entre los primeros ejemplos conservados de la ilustración del pontifical de la Curia romana. A favor de esa precocidad iconográfica se halla el hecho de que, aunque se destaque la presencia del obispo/papa en cada inicial historiada, se otorgue aún un gran protagonismo a los eclesiásticos que reciben el resultado de la acción ritual $\mathrm{y}$, asimismo, la forma de la mitra episcopal, con escaso desarrollo en altura, y, sobre todo, de la tiara papal. No se trata de la triple corona, como será característico a partir del siglo XIV, sino de una tiara cónica, con textura cuadrada en blanco y una cinta de oro en forma de diadema en la parte inferior. Dicha tiara se observa sistemáticamente en las representaciones de los pontífices previos a Bonifacio VIII (1294-1303) y, notablemente, en las de Inocencio III, precisamente responsable de la definición de la tiara como símbolo del poder terrenal del papa ${ }^{55}$. A partir del mandato de Bonifacio VIII, sobre el galón situado en la parte inferior se fue desarrollando una pequeña corona $\mathrm{y}$, progresivamente, se añadieron otras dos, formando en conjunto un trirregno, símbolo de la soberanía sacerdotal, real e imperial del papa; finalmente, ya en Aviñón, posiblemente bajo Clemente VI (1342-1352), el galón y la pequeña corona iniciales fueron sustituidos por una corona de idéntica factura a las otras dos y las tres se dispusieron a igual distancia, dando como resultado la estructura de la tiara pontificia que se mantendría en el futuro ${ }^{56}$.

Incluso en la iconografía de la primera inicial del pontifical de Tui es posible encontrar otro elemento menor que alude a la cronología temprana de este manuscrito: la presencia de un franciscano entre quienes asisten a la tonsura de un clérigo por parte del pontífice (fig. 2). La inclusión de un mendicante en la escena, rasgo poco habitual entre este tipo de pontificales, ha de ponerse en relación con el impulso que experimentó esta Orden en el entorno de la Curia en los últimos años del siglo XIII, coincidiendo, como ya se señaló, con el ascenso al solio papal de Nicolás IV, que era franciscano. Y, asimismo, se explica si tenemos en cuenta la importancia que, al amparo de la pujanza de Asís, alcanzó el franciscanismo en la región umbra y, particularmente, en la miniatura ejecutada en esa zona o bajo su influencia, tal y como se deduce, por ejemplo, de la importante colección de libros corales procedentes del convento de San Domenico de Perugia y hoy conservados en la Biblioteca Augusta de esa ciudad ${ }^{57}$. Por otra parte, esta influencia franciscana en el contexto que produjo el pontifical de Tui justificaría la presencia-además repetida- de san Francisco en las letanías finales (ff. 249v y 256v) cuando no es frecuente que se mencione a este santo entre los pontificales de la Curia editados por Michel Andrieu ${ }^{58}$.

Ello nos remite al tercer aspecto que abunda en la conclusión de que los ejemplares de Tui y Sevilla fueron realizados en la Curia romana a finales del siglo XIII: su contenido. En su magnífico estudio sobre los pontificales conservados en las bibliotecas públicas de Francia, Victor Leroquais proponía como uno de los posibles mecanismos de datación y localización de este tipo de manuscritos el método histórico, consistente en buscar en el texto alusiones a eclesiásticos conocidos, diócesis concretas, santos de especial devoción en un determinado lugar, ... para disponer de información sobre el propietario o el destinatario del volumen, la iglesia en la que se

54 BilotTA, 2009: 64-65.

55 Paravicini Bagliani, 1998: 67-68 y figs. 16, 22, 29, 30 y 31.

56 PARAVicini Bagliani, 1998: 69 y figs. 48, 49, 50, 51 y 61.

57 Véase al respecto PARMEgGiani, 2006.

58 Solo se incluye a san Francisco en las letanías finales del pontifical Ciudad del Vaticano, BAV, ms. Vat. lat. 4745. También se le menciona en otros seis manuscritos, pero en estos casos su nombre ha sido añadido al margen $a$ posteriori (París, BNF, mss. lat. 960 y lat. 15619; Lyon, Bibliothèque Municipale, ms. 5132; y Ciudad del Vaticano, BAV, mss. Vat. lat. 5747, Vat. lat. 5791 y Borghes. lat. 72) (ANDrieu, 1940: 519). 
empleó, el momento en que se fabricó, etc. ${ }^{59}$. Si aplicamos este criterio a los pontificales de Castilla, podemos extraer una primera conclusión: no existe en todo el texto ninguna referencia de estas características; esto es, el contenido de los dos ejemplares está notablemente tipificado, rasgo que comparten con el resto de los pontificales elaborados en los scriptoria de la Curia en unos años en que, tras la reforma litúrgica ya comentada, fue necesario incrementar la producción de manuscritos para abastecer las nuevas necesidades rituales.

El propio contenido de los dos volúmenes es expresivo de este origen: como ya se señaló, pertenecen a la familia de los pontificales de la Curia romana del siglo XIII, aunque no fueron incluidos en la edición realizada por Michel Andrieu ${ }^{60}$. Y, en concreto, de acuerdo con la clasificación realizada por este liturgista, se corresponden con la versión mixta o $\beta$, que es precisamente la misma que contienen algunos de los pontificales estilística e iconográficamente más próximos a nuestros ejemplares (París, BNF, ms. lat. 960, o Ciudad del Vaticano, BAV, mss. lat. 1155 y 4747$)^{61}$.

El rasgo distintivo de los manuscritos de esta versión es que presentan un texto muy similar al de la versión breve o $\alpha$, pero suelen ampliarla o introducir alguna modificación, generalmente a través de pequeñas adiciones marginales, que los aproximan a la versión larga o $\gamma$, sin llegar a su amplitud ${ }^{62}$, síntoma de las oscilaciones que se produjeron en el momento de fijación de estos textos y muestra, por tanto, del carácter temprano de estos manuscritos. Según M. Andrieu, la versión $\alpha$ se caracteriza por la ausencia de los ordines IV, XX-XXI y XXXVIII-XL, por la escritura del ordo XIII según la forma A y del XV según la forma B y por la situación del ordo XXV fuera de su lugar, entre los ordines LIII y LIV. La versión $\gamma$, por su parte, contiene todos los ordines de manera correlativa y, además, el XV está escrito según la forma $\mathrm{B}^{63}$.

En el caso del pontifical de Tui, en su forma inicial se observa, como ocurre en la versión breve, la ausencia de los ordines IV, XX-XXI y XXXVIII-XL, además de que los ordines XIII y XV responden, respectivamente, a las formas A y B. Sin embargo, la situación del ordo XXV no está alterada y, lo que es más importante, en la elaboración del manuscrito intervino una segunda mano, paleográficamente diferenciada de la primera, que añadió varios de los ordines que faltaban en un principio, aproximando, así, el texto a la versión más amplia: los XX-XXI fueron copiados al final de las primeras letanías (ff. 251v-255) -obligando a escribir unas segundas a continuación (ff. 255-258)- y los XXXVIII-XL se incorporaron en el margen del f. 176. El carácter mixto de este manuscrito queda, asimismo, atestiguado por la adición de dos textos que no pertenecen al pontifical de la Curia romana. En los ff. 258v-259, después de la fórmula Explicit Liber Pontificalis. Deo gratias. Amen (f. 258), se añadió un ordo confirmationis prelatorum, compuesto por una serie de versículos, responsorios y oraciones de uso común en la liturgia ${ }^{64}$. Y

59 LeRoquais, 1937: I, CI-CXVI.

${ }^{60}$ Véase la nota 16.

${ }^{61}$ La nómina completa de los pontificales de la versión mixta editados por M. Andrieu es la siguiente: Lyon, Bibliothèque Municipale, ms. 5132; París, BNF, mss. lat. 960, lat. 15619 y lat. 17336; y Ciudad del Vaticano, BAV, mss. lat. 1153, 1155, 4747 y 5771, ms. Borghes. lat. 72 y ms. Vat. Barb. 549 (ANDrieu, 1940: 255-261).

62 Andrieu, 1940: 231

63 ANDRIEU, 1940: 229-231.

${ }^{64}$ Ordo confirmationis prelatorum. Posquam dominus papa electos pronuntiaverit ut moris est. Capellanus ad altare eundo cantent: Te Deum laudamus. Quo cantato antiquorum episcoporum, dicat: Pater noster. Vers.: Et ne nos inducas interpretatione. Resp.: Set libera nos a malo. Salvos fac servos tuos. Resp.: Dominus misericors sperantes inte. Mitte eis Domine auxilium de sancto. Resp.: Et de Syon tuere eos. Vers.: Domine exaudi orationem meam. Resp.: Et clamor meus. Vers.: Dominus vobiscum. Resp.: Et cum spiritu tuo. Oremus: Omnipotens sempiterne Deus miserere famulis tuis electis et dirige eos secundum tuam clementiam in viam salutis eterne ut te donante tibi placita cupiant et tota virtute perficiant (MOELLER, 1992-2004: VI, n. ${ }^{\circ}$ 3859).

Arch. esp. arte, LXXXV, 339, JULIO-SEPTIEMBRE 2012, 213-232, ISSN: 0004-0428 
en el f. 260, al final del volumen, se volvió a copiar el inicio del ritual correspondiente al jueves santo -ya existente en el manuscrito (ff. 177-190)-, desde Incipit ordo romanus qualiter agendum sit quinta feria in cena domini. Statio ad sanctum Iohannem ad Lateranum hasta per indulgentiam subvenitur. Amen, para incluir a continuación (ff. 260-269v) un fragmento del ordo del mismo día pero según la versión del pontifical romano-germánico, compuesto a mediados del siglo $X^{65}$

El hecho de que se trate de un manuscrito elaborado en la Curia, y no en Perugia, pese a la importante influencia de la miniatura umbra en su decoración, se manifiesta incluso, desde el punto de vista del texto, en que tanto en los ff. $248 \mathrm{v}-251 \mathrm{v}$ como en los ff. 255-258 se contienen sendas letanías típicamente romanas, que nada tienen que ver con los santos incluidos en las letanías características de los manuscritos destinados a ser usados en las iglesias de Umbría ${ }^{66}$.

El ejemplar de Sevilla apunta hacia ese mismo origen romano y nos remite a la versión mixta del pontifical de la Curia. Son muchos más numerosos que en el códice de Tui los ordines que no encontramos actualmente en el manuscrito, pero en su mayor parte se debe a la importante pérdida de soporte sufrida ${ }^{67}$. Sin embargo, se puede afirmar con rotundidad que los ordines XX y XXI no existen porque no se copiaron desde el primer momento, como es característico de la versión breve; y, por lo mismo, conviene notar que el ordo XIII responde a la forma A y el XV, a la forma B. En consecuencia, también es posible aventurar que el ordo IV, hoy desaparecido junto a los primeros folios del manuscrito, no existiera ya en la versión original, como es habitual en los pontificales breves. Frente a ello, el pontifical de Sevilla sí contiene los ordines XXXVIII-XL -copiados, además, por la mano principal responsable del manuscrito-, lo que lo aproxima a la versión larga $\mathrm{y}$, por tanto, a falta de una buena parte del texto -incluyendo las letanías-, permite concluir que el contenido de este ejemplar es el característico de la versión mixta, fruto de las oscilaciones inherentes a los momentos iniciales de fijación de este tipo de libros litúrgicos.

La propia materialidad del texto, es decir, la escritura apunta, al igual que los rasgos estilísticos, la iconografía y el contenido, hacia un origen indudablemente situado en la Curia romana a finales del siglo XIII, pues los dos ejemplares que estudiamos están escritos en una gótica libraria italiana, de módulo grande y ligeramente alargado, que es muy similar a la de los demás pontificales producidos en el entorno papal en esta época.

Podemos afirmar, en conclusión, que la conservación de ambos pontificales en sendas instituciones españolas ${ }^{68}$, alejados del grupo de manuscritos al que pertenecieron en origen, ha supuesto un olvido historiográfico notable y, sobre todo, su desconocimiento por parte de los investigadores que, fundamentalmente desde Francia e Italia, han tratado de reconstruir el disperso y malogrado fondo de manuscritos litúrgicos realizados para uso de la capilla papal. Sin embargo, sus rasgos estilísticos, con una fuerte impronta de la miniatura perusina, su iconografía, alusiva al refuerzo de la autoridad papal, su contenido, correspondiente con las versiones más

Alia oratio: Actiones nostras quaesumus Domine aspirando perveni et adiuvando prosequere ut omnis nostra actio et oratio a te semper intipiat (sic) et per te, cepta, finiatur (MoELler, 1992-2004: VI, n. ${ }^{\circ}$ 74). Per Christum Dominum nostrum. Resp.: Amen. Et benedictio Dei patris omnipotentes: Patris et Filii et Spiritu Sancti descendat super eos et maneat semper. Resp.: Amen.

${ }^{65}$ Se trata, en concreto, de un fragmento del ordo XCLX, De officiis divinis a cena Domini usque in octavas pentecostes. Feria quinta maioris ebdomadae, que termina abruptamente como consecuencia de la pérdida de soporte: Sedentem autem pontifice pro foribus... Propitiare ergo, Domine, trementibus atque supplicibus sub sententie tue expectatione et ad humilitatem iacenti<um> (VoGEL, Elze, 1963: II, 59-65).

${ }^{66}$ Véase el listado de santos característicos del calendario perusino de esta época en CALECA, 1969: 172-173.

${ }^{67}$ Se debe a esta razón la inexistencia de los ordines XVI, XXIV-XXVII, XLIV-XLV, XLIX, L y LIV.

${ }^{68}$ Hemos prescindido del análisis, enormemente interesante, de la trayectoria postmedieval de ambos manuscritos, desde Roma hasta sus respectivos lugares de conservación, por superar los límites propuestos para este artículo, pero abordaremos esta cuestión en ulteriores trabajos. 
tempranas del pontifical de la Curia romana, y su escritura, claramente italiana, demuestran, sin lugar a dudas, que, como otros manuscritos conservados en diversas bibliotecas francesas e italianas, los pontificales de Tui y Sevilla fueron realizados en Roma a finales del siglo XIII. Y constituyen, por lo tanto, dos nuevos exponentes de la intensa actividad de producción manuscrita e iluminación llevada a cabo en el entorno de la Curia papal en las décadas centrales del medioevo.

\section{BIBLIOGRAFÍA}

Álvarez Márquez, M. del Carmen, Manuscritos localizados de Pedro Gómez Barroso y Juan de Cervantes, arzobispo de Sevilla (Anexos de "Signo", 3), Alcalá de Henares, Universidad de Alcalá, 1999.

Andrieu, Michel, Le Pontifical romain au Moyen Âge, tomo 2: Le Pontifical de la Curie romaine au XIII ${ }^{e}$ siècle ("Studi e Testi', 87), Ciudad del Vaticano, Biblioteca Apostólica Vaticana, 1940.

Avril, François (dir.), Dix siècles d'enluminure italienne, $V I^{e}-X V I^{e}$ siècles, cat. exp., París, Bibliothèque Nationale de France, 1984.

Avril, François, Gousset, Marie-Thérèse y Rabel, Claudia, Manuscrits enluminés de la Bibliothèque nationale, vol. II: Manuscrits enluminés d'origine italienne, XIII ${ }^{e}$ siècle, París, Bibliothèque Nationale de France, 1984.

Bilotta, Maria Alessandra, "I codici miniati prodotti in Laterano conservati nella Biblioteca Apostolica Vaticana", Miscellanea Bibliothecae Apostolicae Vaticanae, 10 (2003), pp. 7-50.

Bilotta, Maria Alessandra, "Contributi per la storia della produzione miniata ad uso del Papato nel Medioevo: i Pontificali duecenteschi secundum consuetudinem et usum Romanae Curiae", Arte medievale, 7 (2008), pp. 55-80.

Bilotta, Maria Alessandra, "Immagine e memoria liturgica nel manoscritti miniati duecenteschi ad uso della Cappella papale", en Carlo Quintavalle, Arturo (ed.), Medioevo: immagine e memoria, Milán, Electa, 2009, pp. 61-68.

Caleca, Antonino, Miniatura in Umbria. I.- La Biblioteca Capitolare di Perugia, Florencia, Marchi \& Bertolli, 1969.

Ciardi Dupré dal Poggetto, Maria Grazia, "La miniatura del Duecento in Italia centrale", en Putaturo, Antonella y Perriccioli, Alessandra (eds.), La miniatura in Italia, vol. I: Dal tardoantico al Trecento con riferimenti al Medio Oriente e all'Occidente europeo, Nápoles, Edizioni scientifiche italiane, 2005, pp. 194-205.

Conti, Alessandro, La miniatura bolognese. Scuole e botteghe. 1270-1340, Bolonia, Alfa, 1981.

Damongeot, Marie-Françoise y Avril, François, "Les miniatures du pontifical d'Autun", Art de l'enluminure, 35 (diciembre 2010-febrero 2011), pp. 38-71.

De Benedictis, Cristina y Neri Lusanna, Enrica, "Miniatura umbra del Duecento: difussione e influenza a Roma e nell'Italia meridionale", Studi di storia dell'arte, 1 (1990), pp. 9-33.

Domínguez Bordona, Jesús, Manuscritos con pinturas. Notas para un inventario de los conservados en colecciones públicas y particulares de España, Madrid, Blass, 1933.

Folda, Jaroslav, "Manuscripts of the History of Outremer by William of Tyre: a handlist", Scriptorium, 27 (1973), pp. 90-95.

Folda, Jaroslav, Crusader manuscript illumination at Saint-Jean d'Acre, 1274-1291, Princeton, Princeton University Press, 1976.

Goullet, Monique, Lobrichon, Guy y Palazzo, Eric, Le Pontifical de la Curie romaine au XIII siècle, París, Éditions du Cerf, 2004.

Gousset, Marie-Thérèse, "Manoscritti miniati a Roma nei fondi della Bibliothèque Nationale di Parigi", en Righetti Tosti-Croce, Marina (ed.), Bonifacio VIII e il suo tempo: anno 1300, il primo Giubileo, cat. exp., Milán, Electa, 2000, pp. 107-110.

Janini, José, Manuscritos litúrgicos de las bibliotecas de España, Burgos, Aldecoa, 1977, vol. I: Castilla y Navarra.

Arch. esp. arte, LXXXv, 339, JULIO-SEPTIEMBRE 2012, 213-232, ISSN: 0004-0428 
Jullien de Pommerol, Marie-Henriette y Monfrin, Jacques, La bibliothèque pontificale à Avignon et à Peñiscola pendant le Grand Schisme d'Occident et sa dispersion: inventaires et concordances, Roma, École Française de Rome, 1991.

Jullien de Pommerol, Marie-Henriette, "La bibliothèque de Boniface VIII", en Lombardi, Giuseppe y Nebbiai dalla Guarda, Donatella (eds.), Libri, lettori e biblioteche dell'Italia medievale (secoli IX-XV). Fonti, testi, utilizzazione del libro, Roma, Istituto Centrale per il Catalogo Unico delle Biblioteche Italiane, 2001, pp. 487-505.

Kay, Richard, Pontificalia. A repertory of latin manuscript pontificals and benedictionals, Kansas, University of Kansas, 2007, en línea: <http://hdl.handle.net/1808/4406> [Consulta: 22 de septiembre de 2009].

Krautheimer, Richard, Rome: profile of a city, 312-1308, Princeton, Princeton University Press, 1980.

Leroquais, Victor, Les pontificaux manuscrits des bibliothèques publiques de France, París, Macôn, 1937, 4 vols.

López-Mayán, Mercedes, "Perspectivas y problemas del análisis histórico de una sede catedralicia: el ejemplo del Pontifical de Tui", Lope de Barrientos. Seminario de Cultura, 1 (2008), pp. 147-163.

Lunghi, Elvio, "Le miniature nei manoscritti italiani della Biblioteca Capitolare di Perugia (secoli XIII, XIV e XV). Le ultime acquisizioni della critica e alcune considerazioni sulla tradizione perugina della decorazione libraria", en Cianini Pierotti, M. Luisa (ed.), Una città e la sua cattedrale: il Duomo di Perugia, Perugia, Edizioni Chiesa S. Severo a Porta Sole, 1992, pp. 249-276.

Lunghi, Elvio, "Maestro del Messale di Deruta/Maestro dei Corali di Assisi", en Bollati, Milvia (dir.), Dizionario biografico dei miniatori italiani (secoli $I X-X V I$ ), Milán, Sylvestre Bonnard, 2004, pp. 627628.

Manso Porto, Carmen e Yzquierdo Perrín, Ramón, "Pintura y miniatura”, en Rodríguez Iglesias, Francisco (dir.), Galicia. Arte, tomo XI: Arte medieval (II), A Coruña, Hércules, 1993, pp. 484-501.

Marques, Luiz C., La peinture du Duecento en Italie centrale, París, Picard, 1987.

Moeller, Eugène (ed.), Corpus Orationum, Turnhout, Brepols, 1992-2004.

Monfrin, Jacques, "La bibliothèque pontificale au Moyen Âge", en Compte-rendu de la soixante-septième session annuelle du Comité, Bruselas, Union Académique Internationale, 1993, pp. 99-116.

Neri Lusanna, Enrica, "Il miniatore del Messale di Deruta e i corali del San Pietro a Gubbio", en Pirovano, Carlo (ed.), Francesco d'Assisi. Documenti e Archivi. Codici e Biblioteche. Miniature, cat. exp., Milán, Electa, 1982, pp. 178-188.

Pace, Valentino, "Codici miniati a Roma al tempo del primo giubileo", en Fagiolo, Marcello y Madonna, M. Luisa (eds.), Roma 1300-1875. L’arte degli anni santi, cat. exp., Milán, A. Mondadori, 1984, pp. 318322.

Pace, Valentino, "Per la storia della miniatura duecentesca a Roma”, en Bierbrauer, Katharina, Klein, Peter K. y Sauerländer, Willibald (eds.), Studien zur mittelalterlichen Kunst, 800-1250. Festschrift für Florentine Mütherich, Munich, Prestel-Verlag, 1985, pp. 255-262.

Palazzo, Eric, L'Évêque et son image: l'illustration du pontifical au Moyen Âge, Turnhout, Brepols, 1999.

Paravicini Bagliani, Agostino, "La mobilità della Curia Romana nel Duecento: riflessi locali", en Società e istituzioni nell'Italia comunale: l'esempio di Perugia (secoli XII-XIV), Perugia, Deputazione di Storia patria per l'Umbria, 1988.

Paravicini Bagliani, Agostino, La Cour des Papes au XIII e siècle, París, Hachette, 1995.

Paravicini Bagliani, Agostino, Le Chiavi e la Tiara. Immagini e simboli del papato medievale, Roma, Viella, 1998.

Paravicini Bagliani, Agostino, "Le biblioteche curiali duecentesche", en Lombardi, Giuseppe y Nebbiai dalla Guarda, Donatella (eds.), Libri, lettori e biblioteche dell'Italia medievale (secoli IX-XV). Fonti, testi, utilizzazione del libro, Roma, Istituto Centrale per il Catalogo Unico delle Biblioteche Italiane, 2001, pp. 264-275.

Parmeggiani, Claudia (ed.), Canto e colore. I corali di San Domenico di Perugia nella Biblioteca comunale Augusta (XIII-XIV sec.), cat. exp., Perugia, Volumnia, 2006.

Pennington, Kenneth, Pope and Bishops. The Papal Monarchy in the Twelfth and Thirteenth Centuries, Filadelfia, University of Pennsylvania Press, 1984.

Rabel, Claudia, "Pontifical", en Charron, Pascal y Guillouët, Jean-Marie (dirs.), Dictionnaire d'histoire de l'art du Moyen Âge occidental, París, R. Laffont, 2009, pp. 754-755. 
Sicart Giménez, Ángel, Pintura medieval: la miniatura, Santiago de Compostela, Velograf, 1981.

Subbioni, Marina, "La miniatura e le corporazioni a Perugia dal XIII al XVII secolo. 1: Maestri e botteghe nella Perugia del Trecento", en Roncetti, Mario (ed.), "Per buono stato de la citade”: le matricole delle Arti di Perugia, cat. exp., Perugia, Volumnia, 2001, pp. 49-52.

Subbioni, Marina, La miniatura perugina del Trecento. Contributo alla storia della pittura in Umbria nel quattordicesimo secolo, Perugia, Guerra, 2003, 2 vols.

Todini, Filippo, "La miniatura in Umbria nel Duecento e nel Trecento", en Pirovano, Carlo (ed.), Francesco d'Assisi. Documenti e Archivi. Codici e Biblioteche. Miniature, cat. exp., Milán, Electa, 1982, pp. 161170.

Van Dijk, Stephan J. P. y Hazelden Walker, Joan, The origins of the modern Roman liturgy: the liturgy of the papal court and the Franciscan Order in the $13^{\text {th }}$ century, Londres, Newman Press, 1960.

Vogel, Cyrille y Elze, Reinhard, Le Pontifical Romano-Germanique du dixième siècle ("Studi e Testi', 227), Ciudad del Vaticano, Biblioteca Apostolica Vaticana, 1963, 2 vols.

Yarza Luaces, Joaquín, "Manuscritos iluminados boloñeses en España. Siglos XIII y XIV”, en Colomer, José Luis y Serra Desfilis, Amadeo (dirs.), España y Bolonia. Siete siglos de relaciones artísticas y culturales, Madrid, Centro de Estudios Europa Hispánica, 2006, pp. 31-48.

Fecha de recepción: 6-VI-2011

Fecha de aceptación: 15-XI-2011

Arch. esp. arte, LXXXv, 339, JULIO-SEPTIEMBRE 2012, 213-232, ISSN: 0004-0428 